\title{
Single injection of very mild dose botulinum toxin in the vastus lateralis improves testicular spermatogenesis and sperm motility in ageing experimental mice
}

Risna Kanjirassery Radhakrishnan ${ }^{1}$, Sowbarnika Ravichandran ${ }^{1}$, Aishwarya Sukesh', Balamuthu Kadalmani ${ }^{2}$ and Mahesh Kandasamy ${ }^{1,2,3^{*}}$ (i)

\begin{abstract}
Background: Botulinum toxin (BoNT) is a widely used therapeutic agent that blocks the excessive release of acetylcholine at the neuromuscular junction. Previously, repeated intracremasteric injections and slight overdose of BoNT have been reported to induce adverse effects in the testicular parameter of experimental rodents. However, a mild dose of BoNT is highly beneficial against skin ageing, neuromuscular deficits, overactive urinary bladder problems, testicular pain and erectile dysfunctions. Considering the facts, the possible therapeutic benefits of BoNT on the testis might be achieved at a very minimal dosage and via a distal route of action. Therefore, we revisited the effect of BoNT, but with a trace amount injected into the vastus lateralis of the thigh muscle, and analyzed histological parameters of the testis, levels of key antioxidants and sperm parameters in ageing experimental mice.
\end{abstract}

Results: Experimental animals injected with $1 \mathrm{U} / \mathrm{kg}$ bodyweight of BoNT showed enhanced spermatogenesis in association with increased activities of key antioxidants in the testis, leading to enhanced amount of the total sperm count and progressive motility.

Conclusions: This study signifies that a mild intramuscular dose of BoNT can be considered as a potent treatment strategy to manage and prevent male infertility.

Keywords: BoNT, Acetylcholine, Spermatogenesis, Testis, Sperm, Antioxidants

\section{Background}

Acetylcholine $(\mathrm{ACh})$ is a key neuromodulator of the cholinergic system and biochemical regulator of muscle contraction [1]. ACh plays an important role in steroidogenesis and spermatogenesis in the testis [2, 3]. Abnormal ageing, psychological complications, neuropathogenic and altered metabolic conditions that are

\footnotetext{
*Correspondence: pkmahesh5@gmail.com; mahesh.kandasamy@bdu.ac.in ${ }^{1}$ Laboratory of Stem Cells and Neuroregeneration, Department of Animal Science, School of Life Sciences, Bharathidasan University, Tiruchirappalli, Tamil Nadu 620024, India

Full list of author information is available at the end of the article
}

associated with the elevated levels of ACh and enhanced cholinergic signaling have been reported to induce endocrine imbalance, erectile failure and testicular defects leading to male infertility [4-7]. Therefore, blockade of excessive $\mathrm{ACh}$ release can be considered to restore male reproductive physiology that is lost during ageing and various diseases. Botulinum toxins (BoNTs) are a class of fatal proteins, mainly produced by Clostridium botulinum, which causes muscle paralysis as they act by binding presynaptically to high-affinity recognition sites on the cholinergic nerve terminals and effectively prevents the exocytosis of ACh vesicles at the neuromuscular 
junctions [8-10]. While a trace amount of purified BoNT has been identified to yield long-lasting anti-ageing therapeutic benefits and mitigating neuromuscular defects in various illnesses $[8,9,11]$, some toxicological reports indicated that cremasteric injection of BoNT (10-40 U/ $\mathrm{kg}$ ) induces cell death and impairs spermiogenesis in the testis of experimental animals [12, 13]. However, a recent report revealed that intrascrotal injection of BoNT in adult men did not induce any obvious adverse effect [14]. Nevertheless, ample scientific evidence unequivocally points towards the positive episodes of a mild dose of BoNT against various clinical complications including movement disorders, cognitive deficits and behavioural abnormalities [8, 9, 11, 15-17]. Eventually, therapeutic forms of BoNTs have been used as a potent treatment agent against skin ageing, hyperhidrosis, chronic migraine, strabismus, movement disorders, overactive urinary bladder problems, testicular pain and erectile dysfunctions [8, 9, 18-20]. Notably, mild doses of therapeutic BoNTs have been identified to mitigate oxidative stress and facilitate cytoprotection in various tissues including the brain [11,21-23]. Thus, we speculate that the reported adverse effect of BoNT on the testicular parameters in experimental animals might be largely due to slightly high dose at the proximal site and also by repeated injections. Considering the facts, the possible therapeutic benefits of BoNT on the testis might be achieved at its minimal dose and via distal route of action $[11,24]$. Hence, we revisited the effect of BoNT on the histological parameters of the testis, status of key antioxidants, and sperm count and motility in ageing experimental animals. In this study, ageing experimental male mice were injected with a single dose of $1 \mathrm{U}$ BoNT per $\mathrm{Kg}$ bodyweight into the vastus lateralis of the thigh. After four weeks of time interval, experimental mice were sacrificed and histological parameters of spermatogenesis, sperm count and motility were measured in corroboration with biochemical assessments of antioxidant levels in testis.

\section{Results \\ BoNT treatment protects against the ageing-mediated decline in the total sperm count, motility and their morphological defects in experimental mice}

The estimation of the number of sperms revealed a significant increase in the total sperm count in the BoNT treated group compared to the control group (Control: 9,725,000 $\pm 2,421,260$ vs BoNT: $15,037,500 \pm 2,309,897$ ).

The total percentage of the motile sperms (Control: $31.5 \pm 5.2$ vs BoNT: $43.8 \pm 7.8)$ and the percentage of sperms with progressive motility (Control: $21.5 \pm 5.5$ vs BoNT: $37.3 \pm 6.8$ ) were found to be increased in the BoNT treated group when compared to that of the control group (Fig. 1). In the morphological analysis, the number of tailless sperms (Control: $16.5 \pm 4.8$ vs BoNT: $6 \pm 1.5$ ) as well as headless sperms (Control: $13.5 \pm 6.8$ vs BoNT: $5 \pm 2.8$ ) were found to be minimized in the BoNT treated group than that of the control group (Fig. 2). However, no other obvious differences related to the morphology of sperms were noticed between the control and the BoNT treated group.

\section{BoNT treatment promotes the spermatogenic capacity in ageing experimental mice}

The histological examination of testicular cross-sections revealed no significant differences in the diameter of the seminiferous tubules between the control group and BoNT treated group (Control: $21.8 \pm 6$ vs BoNT: $22.6 \pm 2$ ). Also, the diameter of the lumen of the seminiferous tubule was almost similar among both the control and BoNT injected animals (Control: $8.5 \pm 4.8$ vs BoNT: $6.8 \pm 1.9$ ) (Fig. 3). Strikingly, the histological estimates of different cellular entities in the seminiferous tubules revealed a significant increase in the number of primary spermatocytes (Control: $39 \pm 3$ vs BoNT: $49 \pm 6$ ), secondary spermatocytes (Control: $20 \pm 3$ vs BoNT: $28 \pm 2$ ) and elongated spermatids (Control: $50 \pm 14$ vs BoNT: $99 \pm 22)$ in the testis of the BoNT treated group than that of the control group. However, the estimated number of round spermatids (Control: $86 \pm 17$ vs BoNT: $63 \pm 16$ ) was found to be reduced in the testis of the BoNT treated group than the control group (Fig. 4).

\section{BoNT treatment increases the enzymatic activities} of testicular antioxidants in ageing experimental mice In the biochemical assessment of testicular protein extracts, the antioxidant activities of superoxide dismutase (SOD) (Control: $0.67 \pm 0.04$ vs BoNT: $0.76 \pm 0.05$ ), catalase (Control: $0.17 \pm 0.02$ vs BoNT: $0.27 \pm 0.05)$, reduced glutathione $(\mathrm{GSH})$ (Control: 2.6. \pm 0.40 vs BoNT: $3.6 \pm 0.29$ ), and glutathione peroxidase (GPx) (Control: $0.40 \pm 0.004$ vs BoNT: $0.60 \pm 0.01$ ) were found to be significantly increased in the BoNT treated group compared to the control group (Fig. 5).

\section{Discussion}

The present study demonstrates that a trace amount of BoNT injection at the distal intramuscular site in ageing experimental animals significantly improved the morphological and cytological characteristics of the seminiferous tubule, and the quantity and motility of sperms in association with the enhanced activities of testicular antioxidant enzymes. Recently, BoNT has been reported to facilitate increased locomotion, cognitive functions and reduced anxiety-related behaviours in ageing experimental rodents and in humans $[11,23,25,26]$. It has 


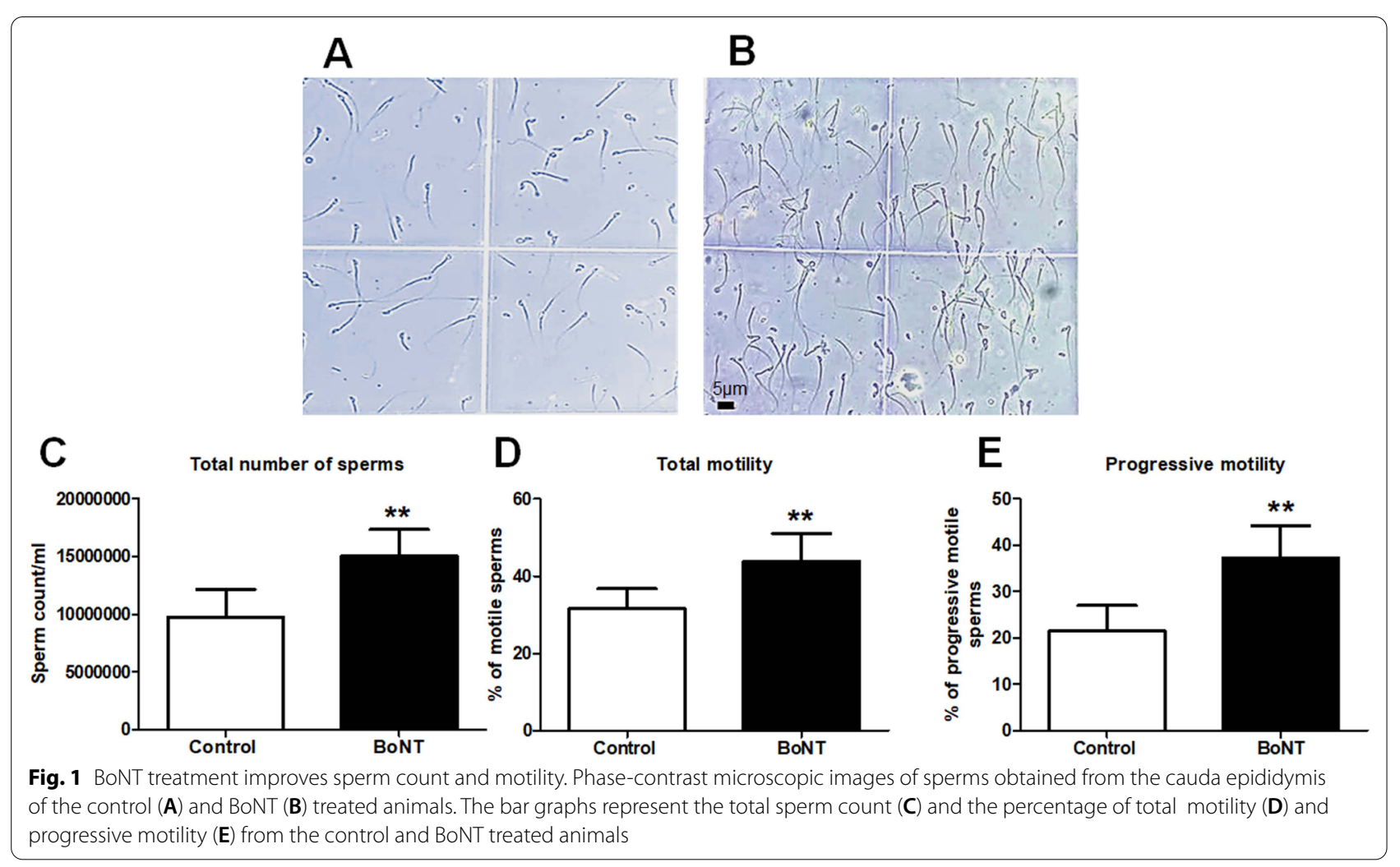

been reported that BoNT treatment improves the blood circulation and oxygen supply, thereby providing trophic support to cell cycle events and cryoprotection in various organs $[27,28]$. Considering the fact, the intramuscular injection of BoNT can be predicted to enhance the blood circulation in the testis of the experimental animals, which can promote the histological parameters and physiological functions of the testis. While the BoNT treated animals showed a significant increase in the number of primary and secondary spermatocytes, and elongated spermatids, a reduction in the number of round spermatids was evident in BoNT treated animals. The latter could be due to the enhanced differentiation of round to elongated spermatids contributing to an enhanced spermiogenesis in BoNT treated group, as BoNT has been reported to facilitate cellular differentiation in different tissues $[29,30]$.

Spermiogenesis is the cellular process of the testis by which haploid round spermatids undergo a series of events to become motile spermatozoa [31, 32]. Spermiogenesis commences after spermatocytes have accomplished the meiotic reductive cycle [33]. This process involves multifaceted morphological, biochemical, and physiological alterations in spermatids [31, 33, 34]. The major events in this process involve embellishment of the acrosome from the Golgi apparatus, condensation of the chromatin, centrosome disintegration, elimination of redundant cytoplasmic portion and formation of flagellum that are responsible for the motility of the spermatozoa $[35,36]$. Thus, the molecular changes associated with BoNT treatment may involve the progression of the flagellum, differentiation of spermatozoa in the testis and increased motility of sperms in the epididymis. Therefore, future studies are required to exactly reveal the possible mechanisms by which BoNT injection facilitates spermatogenesis and sperm motility.

According to the free radical theory of ageing, reactive oxygen species (ROS)-mediated oxidative stress and mitochondrial damage in various tissues and organs represents an underlying biological cause of progressive ageing $[37,38]$. Though generation and maintenance of the optimal levels of ROS can be important for the development and physiological functions of organs, unprecedented increased accumulation of ROS and failure in antioxidant defence would result in dysfunction of organs leading to diseases [37-39]. Among various organs, the testis appears to be highly vulnerable to abrupted levels of ROS [40,41]. Considerable clinical and experimental reports established a clear notion that the high level of ROS could impair steroidogenesis and spermatogenesis in the testis [40]. Oxidative stress has typically been associated with the loss of structural and functional integrity 


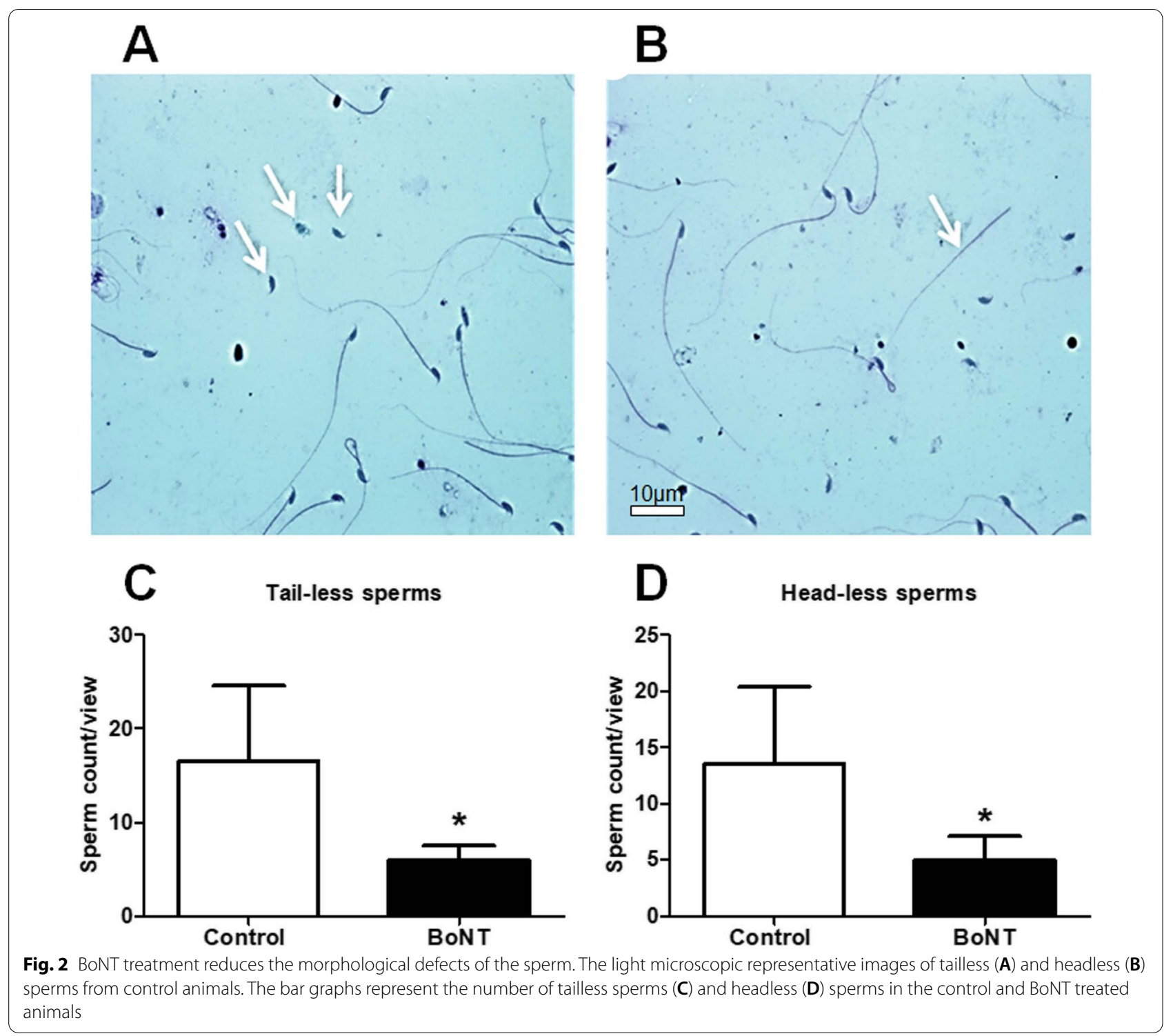

of spermatozoa by inducing damages to DNA, RNA transcripts and telomeres, thereby leading to testicular atrophy and infertility in ageing and disease [38, 42, 43]. Oxidative stress in the testis contributes to low sperm count, impaired motility and abnormal morphology [44, 45]. Several studies have reported that ageing results in excessive production of ROS, reduction in antioxidants and DNA damage in spermatozoa, contributing to poor sperm count and motility [46-49]. Notably, increased levels of ROS has been reported to suppress the circulating concentration of the male sex hormones leading to abnormal hypothalamic-pituitary-gonadal (HPG) axis and infertility related issues [50]. Thus, neutralization of abruptly increased levels of ROS by the implementation of pharmacological agents has been considered as a potent therapeutic regime for infertility in order to restore the testicular functions [51, 52]. Earlier studies have indicated that the supplementation of antioxidants such as vitamin $C$, vitamin $\mathrm{E}$ and glutathione improves spermatogenesis and sperm quality $[53,54]$. Recent studies have demonstrated that the use of therapeutic BoNT reduced oxidative stress in animal models and plasma of patients $[22,55,56]$.

While the physiological levels of $\mathrm{ACh}$ is important for spermatogenesis and sperm motility [57], the abnormal concentration of ACh appears to induce oxidative stress in the testis, leading to and reduction in the motility and functioning of sperms [58, 59]. Notably, administration of $\mathrm{ACh}$ in the inferior spermatic nerve plexus, a site that regulates steroidogenesis, has been reported to block the secretion of testosterone that might be associated with reduced spermatogenesis in the testis [7]. 

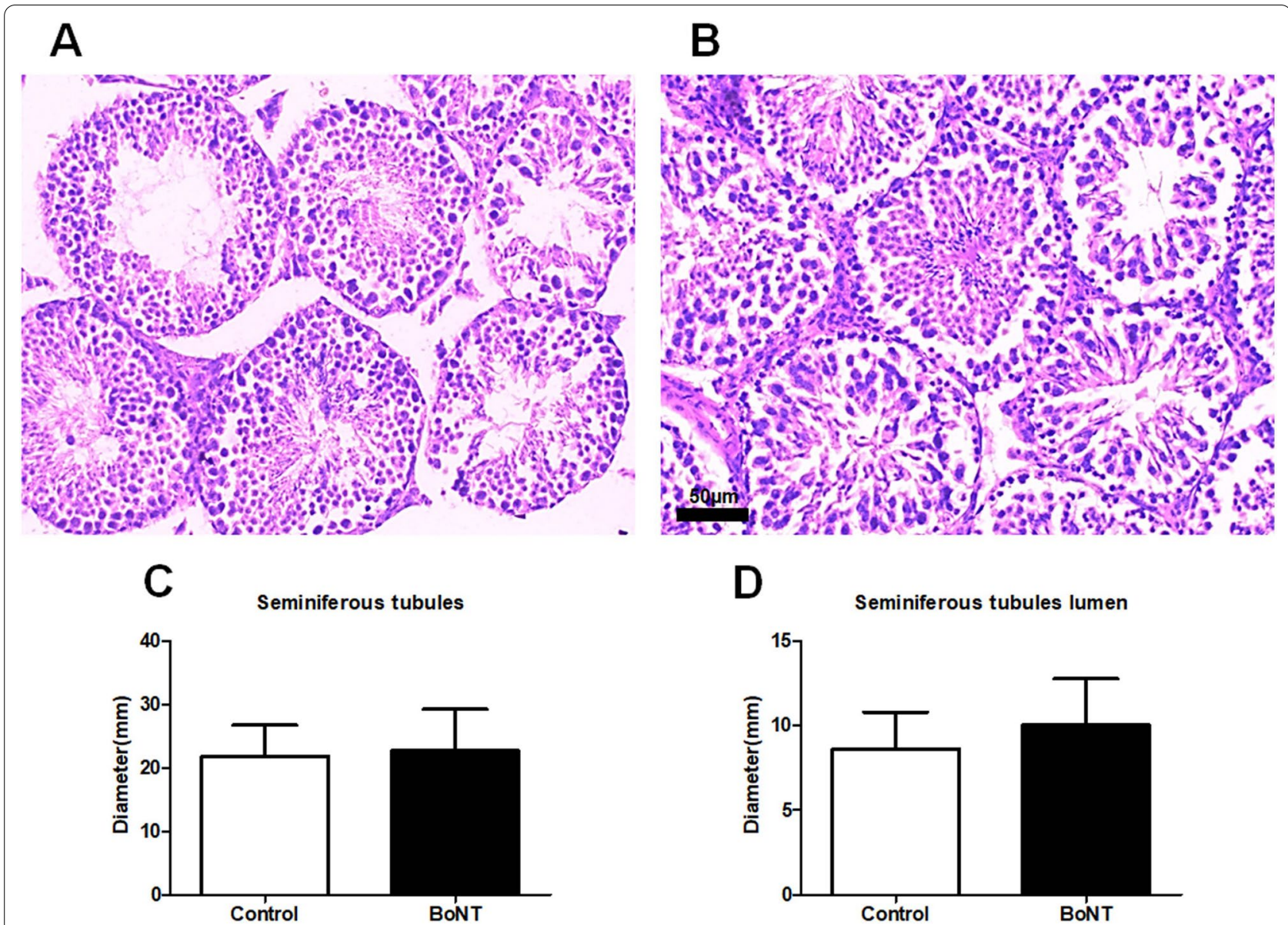

Fig. 3 No major structural difference in the seminiferous tubules between control and BoNT treated animals. The $H$ and $E$ staining represents the morphology of seminiferous tubules in the testis of control (A) and BoNT treated animals (B). The bar graph data represents the diameter of seminiferous tubules $(\mathbf{C})$, the diameter of lumen of seminiferous tubules (D)

Besides, Favaretto et al. reported that abnormal levels of endogenous ACh can interfere with the production of testosterone secretion in rat Leydig cells [60]. Similarly, cholinergic agonists have been reported to mediate inhibition of testosterone biosynthesis in the testis [61]. Therefore, it can be speculated that BoNT treatment might contribute directly to diminish the level of ROS or it may facilitate the production of antioxidants to counteract the free radicals in the testis. Indeed, the anti-ageing properties of BoNT injection in aesthetic rejuvenation and course of action in cosmetics have been considered to be mediated via the suppression of ROS production [62-64]. In corroboration, blood samples derived from the subjects with BoNT injection have been characterized by a reduced level of ROS [55]. Besides, ample reports experimentally validated the cytoprotective role of BoNT as it counterbalances the increased levels of oxidative stress and free radical production with the elevation of antioxidant enzymes. In a study by Uchiyama et al., BoNT has been reported to reduce ROS in vascular endothelial cells in vitro in cutaneous ischemia-reperfusion (I/R) injuries [22], BoNT type B injection has also been shown to reduce oxidative and endoplasmic reticulum stress induced by $I / R$ injury in a mouse model [21]. Yu et al. demonstrated that the level of malondialdehyde (MDA), a marker of oxidative stress which indicates the rate of lipid peroxidation, was considerably reduced in spinal cord injury-induced mice model treated with BoNT along with minocycline, indicating reduced oxidative stress [65]. Further, many studies have suggested the use of therapeutic BoNT-A in urinary bladder dysfunctions resulting from prostatic hyperplasia due to its positive effect in controlling oxidative stress [66, 67]. Very recently, Yesudhas et al. reported that BoNT improves the enzymatic activities of key antioxidants and provides neuroprotection in the brain of ageing experimental animals [11, 23]. Therefore, BoNT might counteract the ageing-associated excessive release of $\mathrm{ACh}$ and regulate 

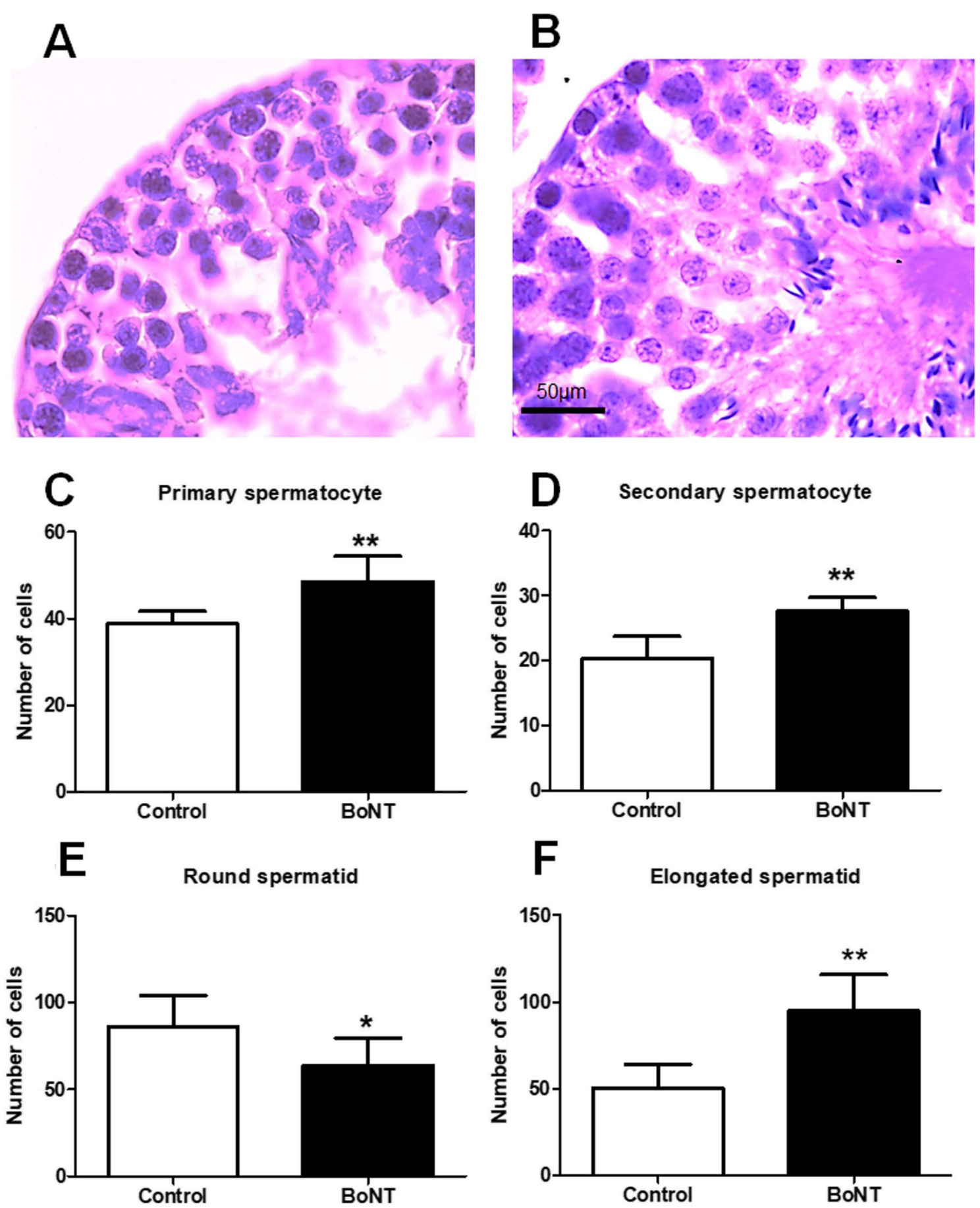

Fig. 4 BoNT treatment indicates increase of spermatogenesis. Light microscopic images of the $\mathrm{H}$ and $\mathrm{E}$ staining depicts the cross sections of the seminiferous tubules in the control (A) and BoNT treated animals (B). The bar graphs show the total number of primary spermatocytes (C), secondary spermatocytes $(\mathbf{D})$, round spermatids $(\mathbf{E})$, and elongated spermatids $(\mathbf{F})$ per cross section of the seminiferous tubule in the testis of control and BoNT treated animals

the production of testosterone and antioxidant enzymes, thereby facilitating spermatogenesis. Moreover, BoNT treatment could potentially elicit cytotropic signalling events or neutralize the apoptotic signalling cascades in the testis, leading to the increased survival of cellular components of the seminiferous tubules. Taken together, a mild amount of BoNT might be a potential therapeutic aid to treat male infertility. 

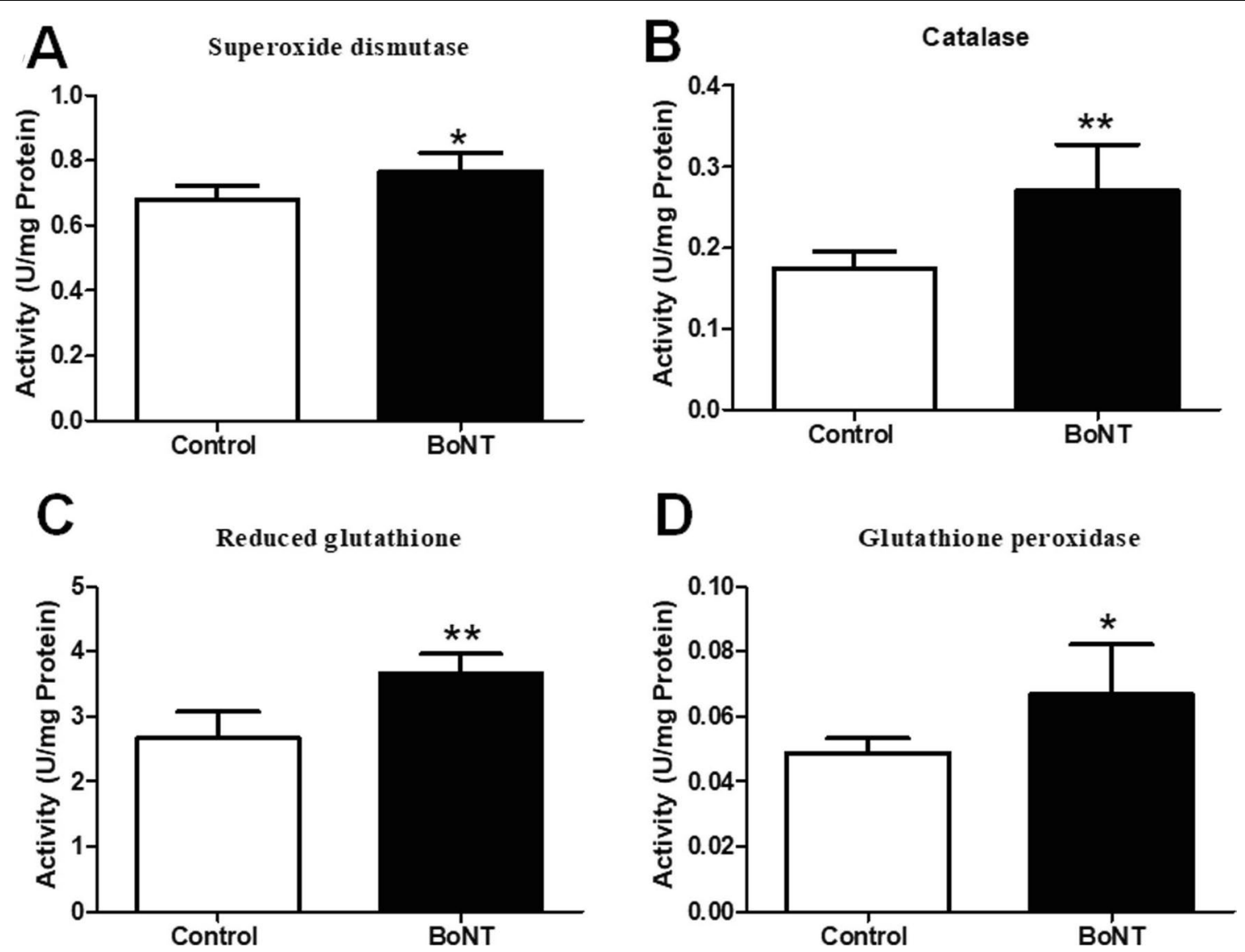

Fig. 5 BoNT treatment increases the activities of key antioxidants in the testis. The bar graph represents the antioxidant activity of SOD (A), catalase $(\mathbf{B})$, reduced glutathione $(\mathbf{C})$ and glutathione peroxidase $(\mathbf{D})$ in $\mathrm{U} / \mathrm{mg}$ protein from the testicular extracts of control and BoNT treated animals

\section{Conclusions}

Reduced sperm count responsible for male infertility has been a rising medical concern worldwide. The risk for male infertility includes ageing, familial, sporadic and abnormal lifestyle, environmental factors, testicular damage and atrophy, infection, exposure to radiation, chemicals, pesticides, hazardous materials, occupational hazards, alcohol consumption, drug addiction, and disorders with metabolic, vascular, neurological, psychiatric and malignancy complications. Though hormonal replacement, aromatase inhibitors, dopamine antagonists and some surgical procedures have been practiced to rectify male infertility, the efficacy of these treatments have been the subject of debate and often they appear to pose adverse effects than clinical rectifications. Of late, cholinergic toxicity has been proposed as an underlying cause of erectile dysfunction, testicular atrophy and infertility. Recently, blockade of ACh release by BoNT has been used to treat various testicular defects like bilateral cremasteric muscle spasms, retractile testis, cryptorchidism and erectile dysfunction. The present study demonstrates that a mild intramuscular injection of BoNT provides defence against ageing-mediated spermatogenic decline and improves the total sperm count and motility in correlation with increased activities of key antioxidants in the testis of experiment mice. Therefore, BoNT might be considered as a potential drug to treat male infertility. However, possibilities for the unknown adverse effects associated with BoNT might not be completely excluded.

\section{Methods}

\section{Injection of BoNT in experimental animals}

For this study, 7-8-months-old $(\mathrm{N}=12)$ male $\mathrm{BALB} / \mathrm{c}$ mice were randomly divided into two groups, namely the control group $(\mathrm{N}=6)$ and BoNT treated group $(\mathrm{N}=6)$. A therapeutic form of BoNT (Allergan, Dublin, Ireland) was dissolved in sterile saline. Experimental mice in the test group have received a single intramuscular injection of BoNT at $1 \mathrm{U}$ per kilogram $(\mathrm{Kg})$ bodyweight $(\mathrm{BW})$ in the vastus lateralis muscle of the thigh as previously described [11,23]. An equal volume of sterile saline was injected to each mouse in the control group. Four weeks 
later, each mouse was sacrificed, the left testis was dissected out and processed for histological examination of spermatogenesis, while the right testis and cauda epididymis were processed for biochemical analysis of antioxidant levels, and sperm analysis respectively. All the experiments were performed in accordance with the approval of the Institutional Animal Ethical Committee (IAEC) under the regulation of the Committee for the Purpose of Control and Supervision of Experimental Animals (CPCSEA), India at Bharathidasan University (Reference No: BDU/IAEC/P272018, Date: 07.08.2018).

\section{Sperm count and motility}

To assess the sperm parameters, the cauda epididymis was removed from each animal and minced in $1 \%$ bovine serum albumin (BSA) prepared with $1 \mathrm{X}$ phosphatebuffered saline (PBS) at $37^{\circ} \mathrm{C}$. The resulting epididymal fluid was diluted in the same solution (1:4) and placed in the Neubauer chamber. The number of sperms and their degree of motility were estimated using an inverted phase-contrast microscope (DMi1, Leica Microsystems, Germany). The total sperm count was calculated using the formula, Sperm count $/ \mathrm{ml}=$ Mean sperm count $x$ dilution factor $\times 10^{4}$. The motility of sperms was graded as progressive and non-progressive motility and total percentage motility was estimated as previously described [68]. Next, smears of the epididymal fluid were prepared on the microscopic slides (Borosil, India) and stained using a solution containing haematoxylin $(\mathrm{H})$ and eosin (E) (Nice Chemicals, India). The $\mathrm{H}$ and E-stained sperms on the slides were fixed and mounted using Dibutylphthalate Polystyrene Xylene (DPX) (Merck, Germany) and dried overnight at room temperature. The morphology and structure of the sperms were evaluated under the light microscope equipped with a camera and the software programme Leica Application Suite (DM750, Leica Microsystems, Germany).

\section{Histological evaluation of seminiferous tubules and spermatogenesis}

The testes were fixed with $10 \%$ neutral buffered formalin (NBF), dehydrated using varying concentrations of ethanol $(70 \%, 80 \%, 90 \%, 100 \%)$ followed by clearing using xylene, after which the tissues were infiltrated and embedded in the paraffin wax. The wax embedded tissues were cut into cross-sections of $10 \mu \mathrm{m}$ thickness using a rotary microtome (Weswox, India). The testicular sections were placed in silane coated microscopic glass slides and stained with $\mathrm{H}$ and $\mathrm{E}$ (Nice Chemicals, India). The histological sections were examined and photodocumented using a light microscope. The total number of the cross-section of seminiferous tubules were counted in 10 non-serial cross-sections of the testis in each animal from the control and treatment group. The diameters of the cross-section of seminiferous tubules and lumens were measured as previously described [69]. Further, the total number of primary spermatocytes, secondary spermatocytes, round spermatids, and elongated spermatids were systematically quantified in seminiferous tubules per animal. The data on the estimated number of cells are represented per seminiferous tubule [70].

Estimation of enzymatic activities of testicular antioxidants The testicular tissue samples were homogenized with radioimmunoprecipitation assay (RIPA) buffer (Thermo scientific) and centrifuged at $12,000 \mathrm{rpm}$ for $20 \mathrm{~min}$ at $4{ }^{\circ} \mathrm{C}$. The supernatant was collected and protein estimation was done by the method described by Lowry et al. [71]. The total protein isolates were subjected to the measurement of key antioxidant enzymes as previously described [11]. In order to measure the activity of SOD, the tissue homogenates were mixed with ice-cold ethanol and chloroform and centrifuged for $15 \mathrm{~min}$ at $12,000 \mathrm{rpm}$ and the supernatants were collected. Further, $0.1 \mathrm{M}$ tris buffer ( $\mathrm{pH}$ 8.2) was added to the supernatant, and the reaction was initiated with the addition of $2.64 \mathrm{mM}$ pyrogallol. As one unit SOD activity represents the amount of protein required for $50 \%$ inhibition of pyrogallol autoxidation per minute, the absorbance was measured at $440 \mathrm{~nm}$ in a microplate reader (Bio-Rad iMark $^{\mathrm{TM}}$ ). To determine the enzymatic strength of catalase, $0.01 \mathrm{M}$ phosphate buffer solution (pH-7.0) and $0.2 \mathrm{M} \mathrm{H}_{2} \mathrm{O}_{2}$ were added to the testicular protein homogenates. After thorough mixing, 5\% potassium dichromate acetic acid reagent was added and the samples were kept in a boiling water bath for $10 \mathrm{~min}$. As the blue colour mixture turn into a greencoloured product of chromate acetate, the absorbance was measured at $570 \mathrm{~nm}$. To measure the activities of $\mathrm{GSH}$, the protein samples were precipitated with $25 \%$ trichloroacetic acid (TCA) and spun down at 3,000 rpm for $10 \mathrm{~min}$ at $4{ }^{\circ} \mathrm{C}$. The supernatant was mixed with $60 \mu \mathrm{M}$ 5,5'-dithiobis-(2-nitrobenzoic acid) (DTNB) and $50 \mathrm{mM}$ potassium phosphate buffer ( $\mathrm{pH}-7.4)$. The absorbance of the resulting yellow-coloured reaction mixture was measured at $412 \mathrm{~nm}$. To assay the activity of GPx, $100 \mu \mathrm{l}$ of protein samples of testes were mixed with a neutral solution containing $0.32 \mathrm{M}$ phosphate, $4 \mathrm{mM} \mathrm{GSH}, 10 \mathrm{mM}$ sodium azide, $2.5 \mathrm{mM} \mathrm{H}_{2} \mathrm{O}_{2}$ and $0.8 \mathrm{mM}$ ethylenediaminetetraacetic acid (EDTA). Then the tubes were incubated for $5 \mathrm{~min}$ at $37{ }^{\circ} \mathrm{C}$ and centrifuged at 3,500 rpm for $15 \mathrm{~min}$. To the supernatant, $0.32 \mathrm{M}$ phosphate solution and $60 \mu \mathrm{M}$ DTNB reagent were added. GSH solutions corresponding to a concentration ranging between 4 and $20 \mu \mathrm{g} / \mathrm{ml}$ were also prepared and served as known standards. The intensity of 
yellow colour developed was measured in a microplate reader at $412 \mathrm{~nm}$. Values were expressed as $\mathrm{mg}$ of GSH consumed per mg of protein [11].

\section{Statistical analysis}

The values are represented as mean \pm standard deviation. Student $t$ test was applied to measure the statistical significance using Graph Pad Prism. The significance level was assumed at $P<0.05$, unless otherwise indicated.

\section{Abbreviations}

ACh: Acetylcholine; BoNT: Botulinum toxin; BW: Body weight; BSA: Bovine serum albumin; CPCSEA: Committee for the Purpose of Control and Supervision of Experimental Animals; DPX: Dibutylphthalate Polystyrene Xylene; DTNB: 5,5'-Dithiobis-(2-nitrobenzoic acid); EDTA: Ethylenediaminetetraacetic acid; GPx: Glutathione peroxidase; GSH: Reduced glutathione; $\mathrm{H}$ and $\mathrm{E}$ : Haematoxylin and eosin; HPG axis: Hypothalamic-pituitary-gonadal axis; IAEC: Institutional Animal Ethical Committee; I/R: Ischemia-reperfusion; MDA: Malondialdehyde; NBF: Neutral buffered formalin; PBS: Phosphate-buffered saline; RIPA: Radioimmunoprecipitation assay buffer; ROS: Reactive oxygen species; SOD: Superoxide dismutase; TCA: Trichloroacetic acid.
\end{abstract}

\section{Acknowledgements}

MK has been supported by the Faculty Recharge Programme, University Grants Commission (UGC-FRP), New Delhi, India. RKR was supported as JRF from the project grant-ECR/2016/000741, SERB, India. BK and MK acknowledge RUSA2.0, Biological Sciences, BDU for the financial support, UGC-SAP, DST-FIST for the infrastructure of the Department of Animal Science, Bharathidasan University. BK would like to thank Teacher Associateship for Research Excellence (TARE), SERB, India (TAR/2020/00027). The authors would like to thank Dr. Muthuswamy Anusuyadevi for helpful advice and support on biochemical assays

\section{Authors' contributions}

MK conceived the idea and design of the study. RKR and BK contributed to further development of the concept and design of the study. RKR, AS, SR and MK were involved in the acquisition of experimental data and analysis. MK and RKR were involved in the initial draft of the manuscript. All authors contributed to the further revision of article, made critical comments and suggestions. All authors read and approved the final manuscript.

\section{Funding}

This work has been supported by a research grant (SERB-EEQ/2016/000639) and an Early Career Research Award (SERB-ECR/2016/000741) from Science and Engineering Research Board (SERB) under the Department of Science and Technology (DST), Government of India.

\section{Declarations}

\section{Availability of data and materials}

The datasets generated during and/or analysed during the current study are available from the corresponding author on reasonable request.

\section{Competing interests}

The authors declare that they have no conflict of interest.

\section{Author details}

${ }^{1}$ Laboratory of Stem Cells and Neuroregeneration, Department of Animal Science, School of Life Sciences, Bharathidasan University, Tiruchirappalli, Tamil Nadu 620024, India. ${ }^{2}$ Department of Animal Science, School of Life Sciences, Bharathidasan University, Tiruchirappalli, Tamil Nadu 620024, India.

${ }^{3}$ Faculty Recharge Programme, University Grants Commission (UGC-FRP), New Delhi 110002, India.
Received: 3 December 2021 Accepted: 23 February 2022

Published online: 04 March 2022

\section{References}

1. Picciotto MR, Higley MJ, Mineur YS. Acetylcholine as a neuromodulator: cholinergic signaling shapes nervous system function and behavior. Neuron. 2012;76(1):116-29.

2. Han X, Zhang C, Ma X, Yan X, Xiong B, Shen W, et al. Muscarinic acetylcholine receptor M5 is involved in spermatogenesis through the modification of cell-cell junctions. Reproduction. 2021;162(1):47-59.

3. Schirmer SU, Eckhardt I, Lau H, Klein J, DeGraafYC, Lips KS, et al. The cholinergic system in rat testis is of non-neuronal origin. Reproduction. 2011;142(1):157-66.

4. Arıcan EY, Gökçeoğlu Kayalı D, Ulus Karaca B, Boran T, Öztürk N, Okyar A, et al. Reproductive effects of subchronic exposure to acetamiprid in male rats. Sci Rep. 2020;10(1):8985.

5. da Silva Júnior ED, de Souza BP, Rodrigues JQD, Caricati-Neto A, Jurkiewicz A, Jurkiewicz NH. Functional characterization of acetylcholine receptors and calcium signaling in rat testicular capsule contraction. Eur J Pharmacol. 2013;714(1-3):405-13.

6. Tata AM, Velluto L, D'Angelo C, Reale M. Cholinergic system dysfunction and neurodegenerative diseases: cause or effect? CNS Neurol Disord Drug Targets. 2014;13(7):1294-303.

7. Zhu C, Palmada MN, Aguado LI, Cavicchia JC. Administration of acetylcholine to the spermatic nerve plexus inhibits testosterone secretion in an in vitro isolated rat testis-nerve plexus system. Int J Androl. 2002;25(3):134-8.

8. Kandasamy M. Perspectives for the use of therapeutic Botulinum toxin as a multifaceted candidate drug to attenuate COVID-19. Med Drug Discov. 2020;6:100042.

9. Nigam PK, Nigam A. Botulinum toxin. Indian J Dermatol. 2010;55(1):8-14.

10. Pirazzini M, Rossetto O, Eleopra R, Montecucco C. Botulinum neurotoxins: biology, pharmacology, and toxicology. Pharmacol Rev. 2017;69(2):200-35.

11. Yesudhas A, Radhakrishnan RK, Sukesh A, Ravichandran S, Manickam N Kandasamy M. BOTOX ${ }^{\circledR}$ counteracts the innate anxiety-related behaviours in correlation with increased activities of key antioxidant enzymes in the hippocampus of ageing experimental mice. Biochem Biophys Res Commun. 2021;569:54-60.

12. Breikaa RM, Mosli HA, Abdel-Naim AB. Influence of Onabotulinumtoxin A on testes of the growing rat. J Biochem Mol Toxicol. 2016;30(12):608-13.

13. Breikaa RM, Mosli HA, Nagy AA, Abdel-Naim AB. Adverse testicular effects of Botox ${ }^{\circledR}$ in mature rats. Toxicol Appl Pharmacol. 2014;275(2):182-8.

14. Ramelli E, Brault N, Tierny C, Atlan M, Cristofari S. Intrascrotal injection of botulinum toxin A, a male genital aesthetic demand: technique and limits. Prog Urol. 2020;30(6):312-7.

15. Cousins E, Ward A, Roffe C, Rimington L, Pandyan A. Does low-dose botulinum toxin help the recovery of arm function when given early after stroke? A phase II randomized controlled pilot study to estimate effect size. Clin Rehabil. 2010;24(6):501-13.

16. Padda IS, Tadi P. Botulinum toxin. Treasure Island: StatPearls Publishing; 2021.

17. Wissel J, Heinen F, Schenkel A, Doll B, Ebersbach G, Müller J, et al. Botulinum toxin $A$ in the management of spastic gait disorders in children and young adults with cerebral palsy: a randomized, double-blind study of "high-dose" versus" "low-dose" treatment. Neuropediatrics. 1999;30:120-4.

18. Ghanem H, Raheem AA, AbdelRahman IFS, Johnson M, Abdel-Raheem T. Botulinum neurotoxin and its potential role in the treatment of erectile dysfunction. Sex Med Rev. 2018;6(1):135-42.

19. Raef HS, Elmariah SB. Treatment of male genital dysesthesia with botulinum toxin. JAAD Case Rep. 2021;10:60-2.

20. Reddy AG, Dick BP, Natale C, Akula KP, Yousif A, Hellstrom WJG. Application of botulinum neurotoxin in male sexual dysfunction: where are we now? Sex Med Rev. 2021:9(2):320-30.

21. Sekiguchi A, Motegi S-I, Uchiyama A, Uehara A, Fujiwara C, Yamazaki $S$, et al. Botulinum toxin B suppresses the pressure ulcer formation in cutaneous ischemia-reperfusion injury mouse model: possible regulation of oxidative and endoplasmic reticulum stress. J Dermatol Sci. 2018;90(2):144-53. 
22. Uchiyama A, Yamada K, Perera B, Ogino S, Yokoyama Y, Takeuchi Y, et al. Protective effect of botulinum toxin $A$ after cutaneous ischemia-reperfusion injury. Sci Rep. 2015;5:9072.

23. Yesudhas A, Roshan SA, Radhakrishnan RK, Abirami GPP, Manickam N,

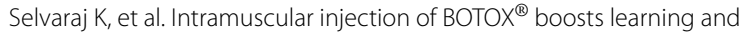
memory in adult mice in association with enriched circulation of platelets and enhanced density of pyramidal neurons in the hippocampus. Neurochem Res. 2020;45(12):2856-67.

24. Kerr G. Safety of botulinum toxin a in cerebral palsy. Toxicon. 2008;51:28.

25. de Jongh R, Bolt I, Schermer M, Olivier B. Botox for the brain: enhancement of cognition, mood and pro-social behavior and blunting of unwanted memories. Neurosci Biobehav Rev. 2008;32(4):760-76.

26. Lewis MB, Bowler PJ. Botulinum toxin cosmetic therapy correlates with a more positive mood. J Cosmet Dermatol. 2009;8(1):24-6.

27. Schweizer DF, Schweizer R, Zhang S, Kamat P, Contaldo C, Rieben R, et al. Botulinum toxin $A$ and $B$ raise blood flow and increase survival of critically ischemic skin flaps. J Surg Res. 2013;184(2):1205-13.

28. Zhibo X, Miaobo Z. Botulinum toxin type A affects cell cycle distribution of fibroblasts derived from hypertrophic scar. J Plast Reconstr Aesthet Surg. 2008;61(9):1128-9.

29. Gugerell A, Kober J, Schmid M, NickI S, Kamolz LP, Keck M. Botulinum toxin $A$ and lidocaine have an impact on adipose-derived stem cells, fibroblasts, and mature adipocytes in vitro. J Plast Reconstr Aesthet Surg 2014:67(9):1276-81

30. Jeong HS, Lee BH, Sung HM, Park SY, Ahn DK, Jung MS, et al. Effect of botulinum toxin type $A$ on differentiation of fibroblasts derived from scar tissue. Plast Reconstr Surg. 2015;136:171e-e178.

31. Linn E, Ghanem L, Bhakta H, Greer C, Avella M. genes regulating spermatogenesis and sperm function associated with rare disorders. Front Cell Dev Biol. 2021;9:634536.

32. O'Donnell L. Mechanisms of spermiogenesis and spermiation and how they are disturbed. Spermatogenesis. 2015;4(2):e979623.

33. Griswold MD. Spermatogenesis: the commitment to meiosis. Physiol Rev. 2016;96(1):1-17.

34. Dadoune JP. The cellular biology of mammalian spermatids: a review. Bull Assoc Anat (Nancy). 1994;78(243):33-40.

35. Berruti G, Paiardi C. Acrosome biogenesis: revisiting old questions to yield new insights. Spermatogenesis. 2011;1(2):95-8.

36. Suphamungmee W, Wanichanon C, Vanichviriyakit R, Sobhon P. Spermiogenesis and chromatin condensation in the common tree shrew, Tupaia glis. Cell Tissue Res. 2008;331(3):687-99.

37. Harman D. Aging: a theory based on free radical and radiation chemistry. J Gerontol. 1956;11(3):298-300.

38. Velusamy T, Panneerselvam AS, Purushottam M, Anusuyadevi M, Pal PK, Jain S, et al. Protective effect of antioxidants on neuronal dysfunction and plasticity in Huntington's disease. Oxid Med Cell Longev. 2017;2017:3279061

39. Imlay JA. Pathways of oxidative damage. Annu Rev Microbiol. 2003:57:395-418

40. Guerriero G, Trocchia S, Abdel-Gawad FK, Ciarcia G. Roles of reactive oxygen species in the spermatogenesis regulation. Front Endocrino (Lausanne). 2014;5:56.

41. Selvaraj K, Manickam N, Kumaran E, Thangadurai K, Elumalai G, Sekar A et al. Deterioration of neuroregenerative plasticity in association with testicular atrophy and dysregulation of the hypothalamic-pituitary-gonadal (HPG) axis in Huntington's disease: a putative role of the huntingtin gene in steroidogenesis. J Steroid Biochem Mol Biol. 2020;197:105526.

42. Aitken RJ, Gibb Z, Baker MA, Drevet J, Gharagozloo P. Causes and consequences of oxidative stress in spermatozoa. Reprod Fertil Dev. 2016;28(1-2):1-10.

43. Sabeti P, Pourmasumi S, Rahiminia T, Akyash F, Talebi AR. Etiologies of sperm oxidative stress. Int J Reprod Biomed. 2016;14(4):231-40.

44. Agarwal A, Virk G, Ong C, du Plessis SS. Effect of oxidative stress on male reproduction. World J Mens Health. 2014;32(1):1-17.

45. Alahmar AT. Role of oxidative stress in male infertility: an updated review. J Hum Reprod Sci. 2019;12(1):4-18.

46. Weir CP, Robaire B. Spermatozoa have decreased antioxidant enzymatic capacity and increased reactive oxygen species production during aging in the Brown Norway rat. J Androl. 2007;28(2):229-40.
47. Mueller A, Hermo L, Robaire B. The effects of aging on the expression of glutathione S-transferases in the testis and epididymis of the Brown Norway rat. J Androl. 1998;19(4):450-65.

48. Luo L, Chen H, Trush MA, Show MD, Anway MD, Zirkin BR. Aging and the brown Norway rat leydig cell antioxidant defense system. J Androl. 2006;27(2):240-7.

49. Liguori I, Russo G, Curcio F, Bulli G, Aran L, Della-Morte D, et al. Oxidative stress, aging, and diseases. Clin Interv Aging. 2018;13:757-72.

50. Darbandi M, Darbandi S, Agarwal A, Sengupta P, Durairajanayagam D, Henkel R, et al. Reactive oxygen species and male reproductive hormones. Reprod Biol Endocrinol. 2018;16(1):87.

51. Asadi N, Bahmani M, Kheradmand A, Rafieian-Kopaei M. The impact of oxidative stress on testicular function and the role of antioxidants in improving it: a review. J Clin Diagn Res. 2017;11(5):IE01-5.

52. Selvaraj K, Ravichandran S, Krishnan S, Radhakrishnan RK, Manickam N, Kandasamy M. Testicular atrophy and hypothalamic pathology in COVID19: possibility of the incidence of male infertility and HPG axis abnormalities. Reprod Sci. 2021;28(10):2735-42.

53. Ahmadi S, Bashiri R, Ghadiri-Anari A, Nadjarzadeh A. Antioxidant supplements and semen parameters: an evidence based review. Int J Reprod Biomed. 2016;14(12):729-36.

54. Majzoub A, Agarwal A. Systematic review of antioxidant types and doses in male infertility: benefits on semen parameters, advanced sperm function, assisted reproduction and live-birth rate. Arab J Urol. 2018:16(1):113-24.

55. Dini E, Mazzucchi S, De Luca C, Cafalli M, Chico L, Lo Gerfo A, et al. Plasma levels of oxidative stress markers, before and after BoNT/A treatment, in chronic migraine. Toxins (Basel). 2019;11(10):608.

56. Zhou Y, Yu S, Zhao J, Feng X, Zhang M, Zhao Z. Effectiveness and safety of botulinum toxin type $A$ in the treatment of androgenetic alopecia. Biomed Res Int. 2020;2020:1501893.

57. Bray C, Son J-H, Meizel S. Acetylcholine causes an increase of intracellular calcium in human sperm. Mol Hum Reprod. 2005;11(12):881-9.

58. Ngoula F, Watcho P, Dongmo M-C, Kenfack A, Kamtchouing P, Tchoumboué J. Effects of pirimiphos-methyl (an organophosphate insecticide) on the fertility of adult male rats. Afr Health Sci. 2007;7(1):3-9.

59. Slimen S, Saloua EF, Najoua G. Oxidative stress and cytotoxic potential of anticholinesterase insecticide, malathion in reproductive toxicology of male adolescent mice after acute exposure. Iran J Basic Med Sci. 2014;17(7):522-30.

60. Favaretto AL, Valença MM, Picanço-Diniz DL, Antunes-Rodrigues JA. Inhibitory role of cholinergic agonists on testosterone secretion by purified rat Leydig cells. Arch Int Physiol Biochim Biophys. 1993;101(6):333-5.

61. Kasson BG, Hsueh AJ. Nicotinic cholinergic agonists inhibit androgen biosynthesis by cultured rat testicular cells. Endocrinology. 1985;117(5):1874-80

62. Satriyasa BK. Botulinum toxin (Botox) A for reducing the appearance of facial wrinkles: a literature review of clinical use and pharmacological aspect. Clin Cosmet Investig Dermatol. 2019;12:223-8.

63. Kattimani V, Tiwari RVC, Gufran K, Wasan B, Shilpa PH, Khader AA. Botulinum toxin application in facial esthetics and recent treatment indications (2013-2018). J Int Soc Prev Community Dent. 2019;9(2):99-105.

64. Ganceviciene R, Liakou Al, Theodoridis A, Makrantonaki E, Zouboulis CC. Skin anti-aging strategies. Dermatoendocrinol. 2012;4(3):308-19.

65. Yu Z, Liu J, Sun L, Wang Y, Meng H. Combination of botulinum toxin and minocycline ameliorates neuropathic pain through antioxidant stress and anti-inflammation via promoting SIRT1 pathway. Front Pharmacol. 2021:11:602417.

66. Chiang BJ, Kuo HC, Liao CH. Can botulinum toxin A still have a role in treatment of lower urinary tract symptoms/benign prostatic hyperplasia through inhibition of chronic prostatic inflammation? Toxins (Basel). 2019;11(9):547.

67. Kuo H-C. Botulinum toxin paves the way for the treatment of functional lower urinary tract dysfunction. Toxins (Basel). 2020;12(6):394.

68. Kanimozhi V, Palanivel K, Kadalmani B, Krikun G, Taylor HS. Apolipoprotein $\mathrm{E}$ induction in syrian hamster testis following tributyltin exposure: a potential mechanism of male infertility. Reprod Sci. 2014;21(8):1006-14.

69. Mehraein F, Negahdar F. Morphometric evaluation of seminiferous tubules in aged mice testes after melatonin administration. Cell J. 2011;13(1):1-4 
70. Nakata H, Wakayama T, Takai Y, Iseki S. Quantitative analysis of the cellular composition in seminiferous tubules in normal and genetically modified infertile mice. J Histochem Cytochem. 2015;63(2):99-113.

71. Lowry OH, Rosebrough NJ, Farr AL, Randall RJ. Protein measurement with the Folin phenol reagent. J Biol Chem. 1951;193(1):265-75.

\section{Publisher's Note}

Springer Nature remains neutral with regard to jurisdictional claims in published maps and institutional affiliations.

- fast, convenient online submission

- thorough peer review by experienced researchers in your field

- rapid publication on acceptance

- support for research data, including large and complex data types

- gold Open Access which fosters wider collaboration and increased citations

- maximum visibility for your research: over $100 \mathrm{M}$ website views per year

At BMC, research is always in progress.

Learn more biomedcentral.com/submissions 